\title{
Similarity method for the study of strong shock waves in magnetogasdynamics
}

\author{
Rajan Arora', Sanjay Yadav ${ }^{1}$ and Mohd Junaid Siddiqui ${ }^{*}$
}

\author{
${ }^{*}$ Correspondence: \\ kjunaidsiddiqui@gmail.com \\ ${ }^{2}$ Department of Mathematics, Zakir \\ Husain Delhi College, University of \\ Delhi, Delhi, Delhi 110002, India \\ Full list of author information is \\ available at the end of the article
}

\begin{abstract}
In this paper, a non-dimensional unsteady adiabatic flow of a plane or cylindrical strong shock wave propagating in plasma is studied. The plasma is assumed to be an ideal gas with infinite electrical conductivity permeated by a transverse magnetic field. A self-similar solution of the problem is obtained in terms of density, velocity and pressure in the presence of magnetic field. We use the method of Lie group invariance to determine the class of self-similar solutions. The arbitrary constants, occurring in the expressions of the generators of the local Lie group of transformations, give rise to different cases of possible solutions with a power law, exponential or logarithmic shock paths. A particular case of the collapse of an imploding shock is worked out in detail. Numerical calculations have been performed to obtain the similarity exponents and the profiles of flow variables. Our results are found in good agreement with the known results. All computational work is performed by using software package MATHEMATICA.
\end{abstract}

Keywords: Lie group; similarity solutions; magnetogasdynamics; shock waves

\section{Introduction}

The spread of shock waves under the control of strong magnetic field is a problem of great interest to researchers in a variety of fields such as nuclear science, geophysics, plasma physics and astrophysics. Hunter [1], Guderley [2], Greifinger and Cole [3] studied the problem of blast wave propagation in homogeneous and inhomogeneous media. Most recently, van Dyke and Guttmann [4], Sharma and Radha [5, 6], Madhumita and Sharma [7], Pandey et al. [8], Sharma and Arora [9], Arora et al. [10, 11] presented high accuracy results and alternative approaches for the investigation of blast wave by using the selfsimilar solutions method. In the same decade, a number of analytical solutions for the blast wave propagations have been obtained by Sachdev [12], Chisnell [13] and Singh et al. [14, 15]. Chisnell [13] provided analytical solutions to the problem of converging shock waves by using the singular points method. Singh et al. [14, 15] used the method of Lie group of transformations to obtain an exact solution for unsteady equation of non-ideal gas and magnetogasdynamics.

The magnetic fields have important roles in a variety of astrophysical situations. Complex filamentary structures in molecular clouds, shapes and the shaping of planetary nebulae, synchrotron radiation from supernova remnants, magnetized stellar winds, galactic winds, gamma-ray bursts, dynamo effects in stars, galaxies and galaxy clusters as well as other interesting problems all involve magnetic fields. When the internal disturbances ac-

C) 2014 Arora et al: licensee Springer. This is an open access article distributed under the terms of the Creative Commons Attribution License (http://creativecommons.org/licenses/by/2.0), which permits unrestricted use, distribution, and reproduction in any medium, provided the original work is properly cited. 
companied by an increase in pressure take place in the central region of a star, a shock wave is formed. It travels from the central region to the periphery and emerges at the surface of the star. In the present paper, we consider the problem of propagation of a onedimensional unsteady flow of an inviscid ideal gas permeated by a transverse magnetic field with infinite electrical conductivity as it approaches the surface of a star. It is assumed that mass density distribution in the medium follows a power law of the radial distance from the point of explosion.

In flows with imploding shocks, conditions of very high temperature and pressure can be produced near the center (axis) of implosion on account of the self-amplifying nature of imploding shocks. As a result of high temperatures attained by gases in motion, the effects of nonequilibrium thermodynamics on the dynamic motion of a converging shock wave can be important.

In this paper, we use the method of Lie group invariance under infinitesimal point transformations [16-18] to study the problem of propagation of strong shock waves in a radiating and electrically conducting gas permeated by a transverse magnetic field. The arbitrary constants, occurring in the expressions for the generators of the local Lie group of transformations, give rise to different cases of possible solutions with a power law, exponential or logarithmic shock paths. The Lie symmetry approach does not necessarily take into account the boundary and initial conditions unless the same are invariant under the change of variable transformations.

\section{Basic equations}

The basic equations describing the one-dimensional unsteady non-planar motion in which the direction of magnetic field is orthogonal to the trajectories of gas particles and electrical conductivity is infinite can be written as follows (Whitham [19]):

$$
\begin{aligned}
& \rho_{t}+\rho u_{x}+u \rho_{x}+m \rho u / x=0, \\
& u_{t}+u u_{x}+\frac{1}{\rho}\left(p_{x}+h_{x}\right)=0, \\
& p_{t}+u p_{x}+\gamma p\left(u_{x}+\frac{m u}{x}\right)=0, \\
& h_{t}+u h_{x}+2 h u_{x}+2 h m u / x=0,
\end{aligned}
$$

where $p$ is the gas pressure, $\rho$ is the density, $u$ is the velocity, $\gamma$ is the constant specific heat ratio, $x$ is the single spatial co-ordinate, $t$ is the time, $h$ is the magnetic pressure defined by $h=\mu H^{2} / 2$ with $\mu$ as magnetic permeability and $H$ is the transverse magnetic field; $m=0$ and 1 correspond to planar and cylindrical symmetry, respectively, and the nonnumeric subscripts denote the partial differentiation with respect to the indicated variable unless stated otherwise. The equation of state is taken to be of the form

$$
p=\rho R T
$$

Now, we consider the motion of a shock front, $x=X(t)$, with the shock speed $V=d X / d t$ propagating into a medium specified by

$$
u=0, \quad p=p_{0}(x), \quad h=h_{0}(x), \quad \rho=\rho_{0}(x),
$$


where $T$ is the temperature, $R$ is the gas constant, $p_{0}(x), h_{0}(x)$ and $\rho_{0}(x)$ are some functions of $x$. The Rankine-Hugoniot jump conditions for the strong shocks are as follows (Whitham [19]):

$$
\begin{aligned}
& u=\frac{2}{\gamma+1} V, \quad \rho=\frac{\gamma+1}{\gamma-1} \rho_{0}(X(t)), \\
& p=\frac{2}{\gamma+1} \rho_{0}(X(t)) V^{2}-\frac{1}{2} \frac{(\gamma+1)^{2}}{(\gamma-1)^{2}} C_{0} \rho_{0}(X(t)) V^{2}, \\
& h=\frac{1}{2} \frac{(\gamma+1)^{2}}{(\gamma-1)^{2}} C_{0} \rho_{0}(X(t)) V^{2},
\end{aligned}
$$

where $C_{0}$ is the shock Cowling number which is defined as $C_{0}=\frac{2 h_{0}}{\rho_{0}} V^{2}$, and the suffix 0 denotes the evaluation of the flow parameters just ahead of the shock.

\section{Similarity analysis by invariance groups}

Here, we suppose that there exists a solution of system (1) along a family of curves, called similarity curves, for which system (1) of partial differential equations reduces to a system of ordinary differential equations; this type of solution is called a similarity solution. In order to obtain the similarity solutions of system (1), we derive its symmetry group such that the system is invariant under this group of transformations. The idea of the calculation is to find a one-parameter infinitesimal group of transformations (see Sharma and Arora [9]):

$$
\begin{array}{ll}
x^{*}=x+\varepsilon \chi(x, t, \rho, u, p, h), & t^{*}=t+\varepsilon \psi(x, t, \rho, u, p, h), \\
u^{*}=u+\varepsilon U(x, t, \rho, u, p, h), & \rho^{*}=\rho+\varepsilon S(x, t, \rho, u, p, h), \\
p^{*}=p+\varepsilon P(x, t, \rho, u, p, h), & h^{*}=h+\varepsilon E(x, t, \rho, u, p, h),
\end{array}
$$

where the generators $\chi, \psi, U, S, P$ and $E$ are to be determined in such a way that system (1) of partial differential equations together with conditions (3) and (4) is invariant with respect to transformations (5); the entity $\varepsilon$ is so small that its square and higher powers may be neglected. The existence of such a group allows the number of independent variables in the problem to be reduced by one, and thereby allowing system (1) to be replaced by a system of ordinary differential equations.

In continuation, we shall use the summation convention and introduce the notation $x_{1}=t, x_{2}=x, u_{1}=\rho, u_{2}=u, u_{3}=p, u_{4}=h$ and $p_{j}^{i}=\frac{\partial u_{i}}{\partial x_{j}}$, where $i=1,2,3,4$ and $j=1,2$.

The system of basic equations (1), which is represented as

$$
F_{k}\left(x_{j}, u_{i}, p_{j}^{i}\right)=0, \quad k=1,2,3,4
$$

is said to be constantly conformally invariant under the infinitesimal group (5) if there exist constants $\alpha_{k r}(k, r=1,2,3,4)$ such that for all smooth surfaces, $u_{i}=u_{i}\left(x_{j}\right)$, we have

$$
\mathrm{L} F_{k}=\alpha_{k r} F_{r}
$$

where $\mathrm{L}$ is the Lie derivative in the direction of the extended vector field

$$
\mathrm{L}=\xi^{j} \frac{\partial}{\partial x_{j}}+\eta^{i} \frac{\partial}{\partial u_{i}}+\beta_{j}^{i} \frac{\partial}{\partial p_{j}^{i}}
$$


with

$$
\xi^{1}=\psi, \quad \xi^{2}=\chi, \quad \eta^{1}=S, \quad \eta^{2}=U, \quad \eta^{3}=P, \quad \eta^{4}=E
$$

and

$$
\beta_{j}^{i}=\frac{\partial \eta^{i}}{\partial x_{j}}+\frac{\partial \eta^{i}}{\partial u_{k}} p_{j}^{k}-\frac{\partial \xi^{l}}{\partial x_{j}} p_{l}^{i}-\frac{\partial \xi^{l}}{\partial u_{n}} p_{l}^{i} p_{j}^{n}
$$

where $l=1,2, j=1,2, i=1,2,3,4, n=1,2,3,4$ and $k=1,2,3,4$. Here, repeated indices imply summation convention and $\beta_{j}^{i}$ is the generalization of the derivative transformation.

System (1) implies

$$
\xi^{j} \frac{\partial F_{k}}{\partial x_{j}}+\eta^{i} \frac{\partial F_{k}}{\partial u_{i}}+\beta_{j}^{i} \frac{\partial F_{k}}{\partial p_{j}^{i}}=\alpha_{k r} F_{r}, \quad k=1,2,3,4, r=1,2,3,4 .
$$

Substitution of $\beta_{j}^{i}$ from (7) into (8) yields a polynomial equations in the $P_{j}^{i}$. Setting the coefficients of $P_{j}^{i}$ and $P_{j}^{i} P_{l}^{k}$ to zero yields a system of first order, linear partial differential equations in the generators $\psi, \chi, S, U, P$ and $E$. This system, which is called the system of determining equations, is given as follows:

$$
\begin{aligned}
& S_{t}+u S_{x}+\rho U_{x}+\frac{m}{x}\left(\rho U+u S-\frac{\rho u \chi}{x}\right)=\alpha_{13} \frac{\gamma m p u}{x}+\alpha_{11} \frac{m \rho u}{x}+\alpha_{14} 2 \frac{h m u}{x}, \\
& S_{\rho}-\psi_{t}-u \psi_{x}=\alpha_{11}, \quad S_{u}-\rho \psi_{x}=\alpha_{12}, \quad S_{p}=\alpha_{13}, \\
& S_{h}=\alpha_{14}, \quad U-\chi_{t}+u S_{\rho}-u \chi_{x}+\rho U_{\rho}=\alpha_{11} u, \\
& S+u S_{u}+\rho\left(U_{u}-\chi_{x}\right)=\alpha_{11} \rho+\alpha_{12} u+\alpha_{13} \gamma p+\alpha_{14} 2 h, \\
& \rho U_{p}+u S_{p}=\alpha_{12} \rho^{-1}+\alpha_{13} u, \quad \rho U_{h}+u S_{h}=\alpha_{14} u+\alpha_{12} \rho^{-1} ; \\
& U_{t}+u U_{x}+\rho^{-1}\left(P_{x}+E_{x}\right)=\alpha_{21} \frac{m \rho}{x}+\alpha_{23} \frac{\gamma p m u}{x}+\alpha_{24} \frac{2 h m u}{x}, \\
& U_{\rho}=\alpha_{21}, \quad U_{u}-\psi_{t}-u \psi_{x}=\alpha_{22}, \\
& U_{h}-\rho^{-1} \psi_{x}=\alpha_{24}, \quad U_{p}-\rho^{-1} \psi_{x}=\alpha_{23}, \\
& U-\chi_{t}+u\left(U_{u}-\chi_{x}\right)+\rho^{-1}\left(P_{u}+E_{u}\right)=\alpha_{21} \rho+\alpha_{22} u+\alpha_{23} \gamma p+\alpha_{24} 2 h, \\
& u U_{\rho}+\rho^{-1}\left(P_{\rho}+E_{\rho}\right)=\alpha_{21} u, \\
& -S \rho^{-2}+u U_{p}+\rho^{-1}\left(P_{p}-\chi_{x}+E_{p}\right)=\alpha_{22} \rho^{-1}+\alpha_{23} u, \\
& -S \rho^{-2}+u U_{h}+\rho^{-1}\left(P_{h}-\chi_{x}\right)=\alpha_{22} \rho^{-1}+\alpha_{24} u ; \\
& P_{t}+u P_{x}+\gamma p U_{x}+m \gamma x^{-1}\left(u P+p U-p u \chi x^{-1}\right) \\
& =\alpha_{31} m \rho u x^{-1}+\alpha_{33} m u x^{-1}+\alpha_{34} 2 h m u / x, \\
& P_{\rho}=\alpha_{31}, \quad P_{u}-\gamma p \psi_{x}=\alpha_{32}, \quad P_{p}-\psi_{t}-u \psi_{x}=\alpha_{33}, \quad P_{h}=\alpha_{34}, \\
& u P_{\rho}+\gamma p U_{\rho}=\alpha_{31} u, \\
& \gamma P+u P_{u}+\gamma p\left(U_{u}-\chi_{x}\right)=\alpha_{31} \rho+\alpha_{32} u+\alpha_{33} \gamma p+\alpha_{34} 2 h, \\
& U-\chi_{t}+\gamma p U_{p}+u P_{p}-u \chi_{x}=\alpha_{32} \rho^{-1}+\alpha_{33} u,
\end{aligned}
$$




$$
\begin{aligned}
& u P_{h}+\gamma p U_{h}=\alpha_{32} \rho^{-1}+\alpha_{34} u ; \\
& E_{t}+u E_{x}+2 h U_{x}-2 h m u x^{-2} \chi+2 h m x^{-1} u+2 u m x^{-1} E \\
& \quad=\alpha_{41} m \rho u x^{-1}+\alpha_{43} m u x^{-1}+\alpha_{44} 2 h m u x^{-1}, \\
& E_{\rho}=\alpha_{41}, \quad E_{h}-\psi_{t}-u \psi_{x}=\alpha_{44}, \quad E_{u}-2 h \psi_{x}=\alpha_{42}, \\
& E_{p}=\alpha_{43}, \quad 2 h U_{\rho}+u E_{\rho}=\alpha_{41} u, \\
& 2 E+2 h U_{u}-2 h \chi_{x}+u E_{u}=\alpha_{41} \rho+\alpha_{42} u+\alpha_{43} \gamma p+\alpha_{44} 2 h, \\
& 2 h U_{p}+u E_{p}=\alpha_{42} \rho^{-1}+\alpha_{43} u, \\
& U-\chi_{t}+2 h U_{h}+u E_{h}-u \chi_{x}=\alpha_{44} u+\alpha_{42} \rho^{-1}, \\
& \psi=\psi(x, t), \quad \chi=\chi(x, t) .
\end{aligned}
$$

Solving the above system of determining equations, we obtain the group of transformations as follows:

$$
\begin{aligned}
& S=\left(\alpha_{11}+a\right) \rho, \\
& U= \begin{cases}\left(\alpha_{22}+a\right) u+k_{1}, & m=0, \\
\left(\alpha_{22}+a\right) u, & m=1,\end{cases} \\
& P=\left(2 \alpha_{22}+\alpha_{11}+3 a\right) p, \\
& \chi= \begin{cases}\left(\alpha_{22}+2 a\right) x+k_{1} t+c, & m=0, \\
\left(\alpha_{22}+2 a\right) x+c, & m=1,\end{cases} \\
& \psi=a t+b,
\end{aligned}
$$

where $\alpha_{11}, \alpha_{22}, a, b, c$, and $k_{1}$ are the arbitrary constants.

\section{Self-similar solutions}

The arbitrary constants, which appear in the expressions for the infinitesimals of the invariant group of transformations, yield different cases of possible solutions as discussed below.

Case I. When $a \neq 0$ and $\alpha_{22}+2 a \neq 0$, the change of variables from $(x, t)$ to $(\bar{x}, \bar{t})$, defined as

$$
\bar{x}=\left\{\begin{array}{ll}
x & \text { if } m=1, \\
x+c\left(\alpha_{22}+2 a\right)^{-1} & \text { if } m=0,
\end{array} \quad \bar{t}=t+\frac{b}{a},\right.
$$

does not change system (1). Thus, rewriting the set of equations (13) in terms of the new variables $\bar{x}$ and $\bar{t}$, and then suppressing the bar sign, we obtain

$$
\begin{aligned}
& S=\left(\alpha_{11}+a\right) \rho, \\
& U= \begin{cases}\left(\alpha_{22}+a\right) u+k_{1}, & m=0, \\
\left(\alpha_{22}+a\right) u, & m=1,\end{cases} \\
& P=\left(2 \alpha_{22}+a_{11}+3 a\right) p, \quad E=\left(\alpha_{44}+a\right) h,
\end{aligned}
$$




$$
\begin{aligned}
& \chi= \begin{cases}\left(\alpha_{22}+2 a\right) x+k_{1} t, & m=0, \\
\left(\alpha_{22}+2 a\right) x, & m=1,\end{cases} \\
& \psi=a t
\end{aligned}
$$

The similarity variable and the form of similarity solutions for $\rho, u, p$ and $h$ readily follow from the invariant surface condition which yields

$$
\psi \rho_{t}+\chi \rho_{x}=S, \quad \psi u_{t}+\chi u_{x}=U, \quad \psi p_{t}+\chi p_{x}=P, \quad \psi h_{t}+\chi h_{x}=E .
$$

The set of equations (16) together with (15) yields on integration the following forms of the flow variables:

$$
\begin{aligned}
& \rho=t^{\left(1+\frac{\alpha_{11}}{\alpha}\right)} \widehat{S}(\xi), \\
& u= \begin{cases}t^{\delta-1} \widehat{U}(\xi) & \text { if } m=1, \\
t^{\delta-1} \widehat{U}(\xi)-k^{*} & \text { if } m=0,\end{cases} \\
& p=t^{\left(2 \delta-1+\frac{\alpha_{11}}{a}\right)} \widehat{P}(\xi) \text {, } \\
& h=t^{\left(1+\frac{\alpha_{44}}{a}\right)} \widehat{E}(\xi),
\end{aligned}
$$

where

$$
k^{*}=\frac{k_{1}}{(\delta-1) a} \quad \text { and } \quad \delta=\frac{\alpha_{22}+2 a}{a} .
$$

The functions $\widehat{S}, \widehat{U}, \widehat{P}$ and $\widehat{E}$ depend only on the dimensionless form of the similarity variable $\xi$, which is determined as follows:

$$
\xi= \begin{cases}\frac{x}{A t^{\delta}} & \text { if } m=1 \\ \frac{x}{A t^{\delta}}+\frac{k^{*} t^{(1-\delta)}}{A} & \text { if } m=0\end{cases}
$$

where $A$ is a dimensional constant whose dimensions are obtained by the similarity exponent $\delta$. Since the shock must be a similarity curve, it may be normalized to be at $\xi=1$. The shock path $X$ and the shock velocity $V$ are, then, given by

$$
\begin{aligned}
& X= \begin{cases}A t^{\delta} & \text { if } m=1, \\
A t\left[t^{(\delta-1)}-\frac{k^{*}}{A}\right] & \text { if } m=0,\end{cases} \\
& V= \begin{cases}\frac{\delta X}{t} & \text { if } m=1, \\
A \delta t^{(\delta-1)}-k^{*} & \text { if } m=0 .\end{cases}
\end{aligned}
$$

At the shock, we have the following conditions on the functions $\widehat{S}, \widehat{U}, \widehat{P}$ and $\widehat{E}$ :

$$
\begin{aligned}
& \left.\rho\right|_{\xi=1}=t^{\left(1+\frac{\alpha_{11}}{a}\right) \widehat{S}(1),} \\
& \left.u\right|_{\xi=1}= \begin{cases}t^{\delta-1} \widehat{U}(1) & \text { if } m=1, \\
t^{\delta-1} \widehat{U}(1)-k^{*} & \text { if } m=0,\end{cases} \\
& \left.p\right|_{\xi=1}=t^{2 \delta-1+\frac{\alpha_{11}}{a}} \widehat{P}(1), \\
& \left.h\right|_{\xi=1}=t^{\left(1+\frac{\alpha_{44}}{a}\right)} \widehat{E}(1) .
\end{aligned}
$$


Equations (22), in view of the invariance of jump conditions, suggest the following forms of $\rho_{0}(x)$ and $h_{0}(x)$ :

$$
\begin{aligned}
& \rho_{0}(x)=\rho_{c}\left(x / x_{0}\right)^{\theta}, \\
& h_{0}(x)=h_{c}\left(x / x_{0}\right)^{\mu},
\end{aligned}
$$

and the following conditions on the functions $\widehat{S}, \widehat{U}, \widehat{P}$ and $\widehat{E}$ at the shock:

$$
\begin{aligned}
& \widehat{U}(1)=\frac{2 \delta A}{\gamma+1}, \\
& \widehat{E}(1)=\frac{1}{2} \frac{(\gamma+1)^{2}}{(\gamma-1)^{2}} C_{0} \frac{\rho_{0} h_{c} A^{\mu}}{h_{0} x_{0}^{\mu}}, \\
& \widehat{P}(1)=\frac{2 \rho_{c} \delta^{2} A^{2+\theta}}{(\gamma+1) x_{0}^{\theta}}-\frac{1}{2} \frac{(\gamma+1)^{2}}{(\gamma-1)^{2}} C_{0} \frac{\rho_{c} \delta^{2} A^{2+\theta}}{x_{0}^{\theta}}, \\
& \widehat{S}(1)=\frac{\gamma+1}{\gamma-1} \rho_{c} \frac{A^{\theta}}{x_{0}^{\theta}}
\end{aligned}
$$

where

$$
\begin{aligned}
& \mu=\frac{\alpha_{44}+a}{\alpha_{22}+2 a}, \\
& \theta=\frac{\alpha_{11}+a}{\delta a}, \quad \delta=\frac{\alpha_{22}+2 a}{a},
\end{aligned}
$$

where $\rho_{c}$ and $h_{c}$ are some reference constants associated with the medium.

Using (24), we rewrite equations (17) as follows:

$$
\begin{aligned}
& \rho=\rho_{0}(X(t)) S^{*}(\xi), \quad u=V U^{*}(\xi), \\
& p=\rho_{0}(X(t)) V^{2} P^{*}(\xi), \quad h=\rho_{0}(X(t)) V^{2} E^{*}(\xi) .
\end{aligned}
$$

Substituting (27) in system (1), we obtain the following system of ordinary differential equations in $S^{*}, U^{*}, P^{*}$ and $E^{*}$, which on suppressing the asterisk sign becomes:

$$
\begin{aligned}
& (U-\xi) S^{\prime}+S\left(\theta+U^{\prime}+\frac{m U}{\xi}\right)=0, \\
& (\delta-1) \delta^{-1} U+(U-\xi) U^{\prime}+S^{-1}\left(P^{\prime}+E^{\prime}\right)=0, \\
& 2(\delta-1) \delta^{-1} P+(U-\xi) P^{\prime}+\theta P+\gamma P\left(U^{\prime}+\frac{m U}{\xi}\right)=0, \\
& 2(\delta-1) \delta^{-1} E+\left(\theta+2 m U \xi^{-1}+2 U^{\prime}\right) E+(U-\xi) E^{\prime}=0 .
\end{aligned}
$$

The jump conditions for the strong shock are:

$$
\begin{aligned}
& U(1)=\frac{2}{\gamma+1}, \quad S(1)=\frac{\gamma+1}{\gamma-1}, \\
& P(1)=\frac{2}{\gamma+1}-\frac{1}{2} \frac{(\gamma+1)^{2}}{(\gamma-1)^{2}} C_{0}, \quad E(1)=\frac{1}{2} \frac{(\gamma+1)^{2}}{(\gamma-1)^{2}} C_{0} .
\end{aligned}
$$


Case II. When $a=0$ and $\alpha_{22} \neq 0$, the change of variables from $(x, t)$ to $(\bar{x}, \bar{t})$ is defined as

$$
\bar{x}=x+c\left(\alpha_{22}\right)^{-1}, \quad \bar{t}=t,
$$

the basic equations in system (1) remain unchanged. The form of similarity solutions for the flow variables readily follows from (13), and can be expressed in the following form on suppressing the bar sign:

$$
\begin{aligned}
& \rho=\rho_{0}(X(t)) S^{*}(\xi), \quad u=V U^{*}(\xi), \\
& p=\rho_{0}(X(t)) V^{2} P^{*}(\xi), \quad h=\rho_{0}(X(t)) V^{2} E^{*}(\xi),
\end{aligned}
$$

together with the initial density and initial magnetic pressure:

$$
\rho_{0}(x)=\rho_{c}\left(\frac{x}{x_{0}}\right)^{\theta}, \quad h_{0}(x)=h_{c}\left(\frac{x}{x_{0}}\right)^{\mu}
$$

where $\xi$ is the dimensionless similarity variable, $X(t)$ is the shock location and $V$ is the shock velocity given by

$$
\xi=\frac{x}{x_{0}} e^{-\frac{\delta t}{A}}, \quad X=x_{0} e^{\frac{\delta t}{A}}, \quad V=\frac{\delta x_{0}}{A} e^{\frac{\delta t}{A}},
$$

with $A$ as a dimensional constant. Substituting (30) in the equations in system (1) and using (31), we obtain the following system of ordinary differential equations in $S^{*}, U^{*}, P^{*}$ and $E^{*}$, which on suppressing the asterisk sign becomes:

$$
\begin{aligned}
& (U-\xi) S^{\prime}+S\left(\theta+U^{\prime}+m U \xi^{-1}\right)=0 \\
& U+(U-\xi) U^{\prime}+S^{-1}\left(P^{\prime}+E^{\prime}\right)=0 \\
& (2+\theta) P+(U-\xi) P^{\prime}+\gamma P\left(U^{\prime}+m U \xi^{-1}\right)=0 \\
& \left(2+\theta+2 U^{\prime}+2 m U \xi^{-1}\right) E+(U-\xi) E^{\prime}=0
\end{aligned}
$$

The jump conditions are

$$
\begin{array}{ll}
U(1)= \begin{cases}\frac{2}{\gamma+1} & \text { if } m=1, \\
\frac{2}{\gamma+1}+\frac{k^{*}}{V} & \text { if } m=0,\end{cases} & S(1)=\frac{\gamma+1}{\gamma-1}, \\
P(1)=\frac{2}{\gamma+1}-\frac{1}{2} \frac{(\gamma+1)^{2}}{(\gamma-1)^{2}} C_{0}, & E(1)=\frac{1}{2} \frac{(\gamma+1)^{2}}{(\gamma-1)^{2}} C_{0} .
\end{array}
$$

Case III. When $Q_{*}$ and $\alpha_{22}+2 a=0$, the study reveals that this condition cannot be obtained in an axially symmetric $(m=1)$ flow as it does not permit for the existence of a similarity solution in such a flow pattern. However, this condition can arise in a plane $(m=0)$ flow where the change of variables from $(x, t)$ to $(\bar{x}, \bar{t})$, defined as

$$
\bar{x}=x, \quad \bar{t}=t+\frac{b}{a},
$$


does not change the basic equations in system (1). Accordingly, the similarity variable and the similarity solutions for the flow variables readily follow from (13), and can be expressed in the following form on suppressing the bar sign:

$$
\begin{array}{ll}
\rho=\rho_{0}(X(t)) S^{*}(\xi), & u=V U^{*}(\xi), \\
p=\rho_{0}(X(t)) V^{2} P^{*}(\xi), & h=\rho_{0}(X(t)) V^{2} E^{*}(\xi), \\
\xi=\frac{\left[x-x_{0} \delta \ln \left(\frac{t}{A}\right)\right]}{x_{0}}, & X=x_{0} \delta \ln \left(\frac{t}{A}\right), \quad V=\frac{\delta x_{0}}{t}, \\
\rho_{0}(x)=\rho_{c} e^{\theta\left(\frac{x}{x_{0}}\right)}, \quad h_{0}(x)=h_{c}\left(\frac{x}{x_{0}}\right)^{\mu x} .
\end{array}
$$

Substituting (34), (35) and (36) in the equations in system (1), we obtain the following system of ordinary differential equations in $S^{*}, U^{*}, P^{*}$ and $E^{*}$, which on suppressing the asterisk sign becomes:

$$
\begin{aligned}
& (U-1) S^{\prime}+\theta S+S U^{\prime}=0 \\
& (U-1) U^{\prime}-\frac{U}{\delta}+S^{-1}\left(P^{\prime}+E^{\prime}\right)=0 \\
& (U-1) P^{\prime}+\left(\theta-\frac{2}{\delta}\right) P+\gamma P U^{\prime}=0 \\
& (U-1) E^{\prime}+\left(\theta-\frac{2}{\delta}+2 U^{\prime}\right) E=0 .
\end{aligned}
$$

The jump conditions are:

$$
\begin{aligned}
& U(0)=\frac{2}{\gamma+1}, \quad S(0)=\frac{\gamma+1}{\gamma-1}, \quad P(0)=\frac{2}{\gamma+1}-\frac{1}{2} \frac{(\gamma+1)^{2}}{(\gamma-1)^{2}} C_{0}, \\
& E(0)=\frac{1}{2} \frac{(\gamma+1)^{2}}{(\gamma-1)^{2}} C_{0} .
\end{aligned}
$$

Case IV. When $a=0$ and $\alpha_{22}=0$, this situation is similar to the previous case in the sense that it does not permit for the existence of a self-similar solutions in an axially symmetric flow. However, the plane flow involving a shock wave moving at constant speed admits a self-similar solution. Accordingly, the similarity variable and the similarity solutions for the flow variables follow from (13), and can be expressed in the following form:

$$
\begin{aligned}
& \rho=\rho_{0}(X(t)) S^{*}(\xi), \quad u=V U^{*}(\xi), \\
& p=\rho_{0}(X(t)) V^{2} P^{*}(\xi), \quad h=\rho_{0}(X(t)) V^{2} E^{*}(\xi), \\
& \xi=\frac{\left[x-\frac{x_{0} \delta t}{A}\right]}{x_{0}}, \quad X=x_{0}\left(1+\frac{\delta t}{A}\right), \quad V=\frac{x_{0} \delta}{A}, \\
& \rho_{0}(x)=\rho_{c} e^{\theta \frac{x-x_{0}}{x_{0}}}, \quad h_{0}(x)=h_{c} e^{\mu \frac{x-x_{0}}{x_{0}}} .
\end{aligned}
$$

Substituting (39) in the equations in system (1) for $m=0$, and using (2), we obtain the following system of ordinary differential equations in $S^{*}, U^{*}, P^{*}$ and $E^{*}$, which on sup- 
pressing the asterisk sign becomes:

$$
\begin{aligned}
& (U-1) S^{\prime}+\theta S+S U^{\prime}=0 \\
& (U-1) U^{\prime}+S^{-1}\left(P^{\prime}+E^{\prime}\right)=0 \\
& (U-1) P^{\prime}+\theta P+\gamma P U^{\prime}=0 \\
& (U-1) E^{\prime}+\left(\theta+2 U^{\prime}\right) E=0
\end{aligned}
$$

The jump conditions are:

$$
\begin{aligned}
& U(1)=\frac{2}{\gamma+1}, \quad S(1)=\frac{\gamma+1}{\gamma-1}, \quad P(1)=\frac{2}{\gamma+1}-\frac{1}{2} \frac{(\gamma+1)^{2}}{(\gamma-1)^{2}} C_{0}, \\
& E(1)=\frac{1}{2} \frac{(\gamma+1)^{2}}{(\gamma-1)^{2}} C_{0} .
\end{aligned}
$$

\section{Imploding shocks}

Here, we consider Case I of an imploding strong shock in the neighborhood of implosion. For the problem of a converging shock collapsing at the axis, the origin of time $t$ is taken to be the instant at which the shock reaches the axis so that $t \leq 0$ in (28). Therefore, the definition of the similarity variable is slightly modified by setting

$$
X=A(-t)^{\delta}, \quad \xi=x / A(-t)^{\delta},
$$

so that the intervals of the variables are $-\infty<t \leq 0, X \leq x<\infty$ and $1 \leq \xi<\infty$. At the instant of collapse $(t=0)$, the gas velocity, pressure, density and the sound speed at any finite axial distance $x$ are bounded, but with $t=0$ and finite $x, \xi=\infty$. In order for the quantities $u, p, \rho$ and $h$ to be bounded when $t=0$ and $x$ is finite, we must have the following boundary conditions at $\xi=\infty$ :

$$
U(\infty)=0, \quad \frac{P(\infty)}{S(\infty)}=0, \quad E(\infty)=0
$$

In the matrix notation, system (28) can be written as

$$
C W^{\prime}=B
$$

where $W=(U, S, P, E)^{t r}$, and the matrix $C$ and the column vector $B$ can be identified by observing system (28). In system (28), there is an unknown parameter $\delta$, which cannot be obtained from an energy balance or the dimensional considerations; it is computed only by solving a non-linear eigenvalue problem for a system of ordinary differential equations. The range of similarity variable is $1 \leq \xi<\infty$ for the implosion problem, and system (44) can be solved for the derivatives $U^{\prime}, S^{\prime}, P^{\prime}$ and $E^{\prime}$ in the following form:

$$
U^{\prime}=\frac{\Delta_{1}}{\Delta}, \quad S^{\prime}=\frac{\Delta_{2}}{\Delta}, \quad P^{\prime}=\frac{\Delta_{3}}{\Delta}, \quad E^{\prime}=\frac{\Delta_{4}}{\Delta},
$$

where $\Delta$, defined as the determinant of the matrix $C$, is given by

$$
\Delta=(U-\xi)^{2}\left[(U-\xi)^{2}-\frac{(\gamma P+2 E)}{S}\right]
$$


and $\Delta_{k}(k=1,2,3,4)$ are the determinants obtained from $\Delta$ by replacing the $k$ th column by the column vector $B$. It can be observed that $U<\xi$ in the interval $[1, \infty)$, while $\Delta$ is positive at $\xi=1$ and negative at $\xi=\infty$ indicating that there exists a $\xi \in[1, \infty)$ at which $\Delta$ vanishes, and consequently the solutions become singular. In order to get a non-singular solution of $(44)$ in the interval $[1, \infty)$, we select the value of the exponent $\delta$ such that $\Delta$ vanishes only at the points where the determinant $\Delta_{1}$ is zero too. It can be checked that at points where $\Delta$ and $\Delta_{1}$ vanish, the determinants $\Delta_{2}, \Delta_{3}$ and $\Delta_{4}$ also vanish simultaneously. To find the exponent $\delta$ in such a manner, we introduce the variable $Z$ as follows:

$$
Z(\xi)=(U-\xi)^{2}-\frac{\gamma P(\xi)+2 E(\xi)}{S(\xi)} .
$$

This, in view of (45), implies

$$
Z^{\prime}=\left\{2(U-\xi)\left(\Delta_{1}-\Delta\right)-\frac{\gamma \Delta_{3}+2 \Delta_{4}}{S}+\frac{\gamma P+2 E}{S^{2}} \Delta_{2}\right\} / \Delta .
$$

Equations (45), in view of (48), become

$$
\begin{array}{ll}
\frac{d U}{d Z}=\frac{\Delta_{1}}{\Delta_{5}}, & \frac{d S}{d Z}=\frac{\Delta_{2}}{\Delta_{5}}, \\
\frac{d P}{d Z}=\frac{\Delta_{3}}{\Delta_{5}}, & \frac{d E}{d Z}=\frac{\Delta_{4}}{\Delta_{5}},
\end{array}
$$

where $\Delta_{5}=2(U-\xi)\left(\Delta_{1}-\Delta\right)-\frac{\gamma \Delta_{3}+2 \Delta_{4}}{S}+\frac{\gamma P+2 E}{S^{2}} \Delta_{2}$, with $\xi=U+\left\{Z+\frac{\gamma P+2 E}{S}\right\}^{1 / 2}$.

\section{Numerical results and discussion}

We integrate equations (45) from the shock $Z=Z(1)$ to the singular point $Z=0$ by choosing a trial value of $\delta$, and compute the values of $U, S, P, E$ and $\Delta_{1}$ at $Z=0$; the value of $\delta$ is corrected by successive approximations in such a way that for these values, the determinant $\Delta_{1}$ vanishes at $Z=0$. The values of the similarity exponent $\delta$, obtained from the numerical calculations for different values of $C_{0}, m$ and $\theta$ are given in Table 1 .

It may be noticed that the magnetic field effects enter through the parameter $C_{0}$. The values of the flow variables before collapse and at the instant of collapse are depicted in Figures 1 and 2 .

Table 1 Similarity exponent $\delta$ for planar and cylindrically symmetric flows and the density exponent $\theta$ with $\gamma=1.4$

\begin{tabular}{llllll}
\hline $\boldsymbol{M}$ & $\boldsymbol{\theta}$ & $\boldsymbol{C}_{\mathbf{0}}$ & Computed $\boldsymbol{\delta}$ & $\begin{array}{l}\text { Guderley } \\
\text { [2] } \boldsymbol{\delta}\end{array}$ & \% Error \\
\hline 1 & 0.5 & 0.00 & 0.72855 & 0.74000 & $1.54 \%$ \\
1 & 0.5 & 0.02 & 0.72810 & 0.75000 & $2.92 \%$ \\
1 & 0.5 & 0.05 & 0.85610 & 0.81000 & $5.69 \%$ \\
1 & 1 & 0.00 & 0.64108 & 0.65000 & $1.37 \%$ \\
1 & 1 & 0.02 & 0.64105 & 0.65310 & $1.85 \%$ \\
1 & 1 & 0.05 & 0.64098 & 0.65510 & $2.16 \%$ \\
0 & 1 & 0.00 & 0.75675 & 0.71400 & $5.99 \%$ \\
0 & 1 & 0.02 & 0.75748 & 0.71100 & $6.54 \%$ \\
0 & 1 & 0.05 & 0.75869 & 0.75000 & $1.16 \%$ \\
0 & 0.5 & 0.00 & 0.85382 & 0.82500 & $3.49 \%$ \\
0 & 0.5 & 0.02 & 0.85428 & 0.80350 & $6.32 \%$ \\
0 & 0.5 & 0.05 & 0.85500 & 0.86000 & $0.58 \%$ \\
\hline
\end{tabular}




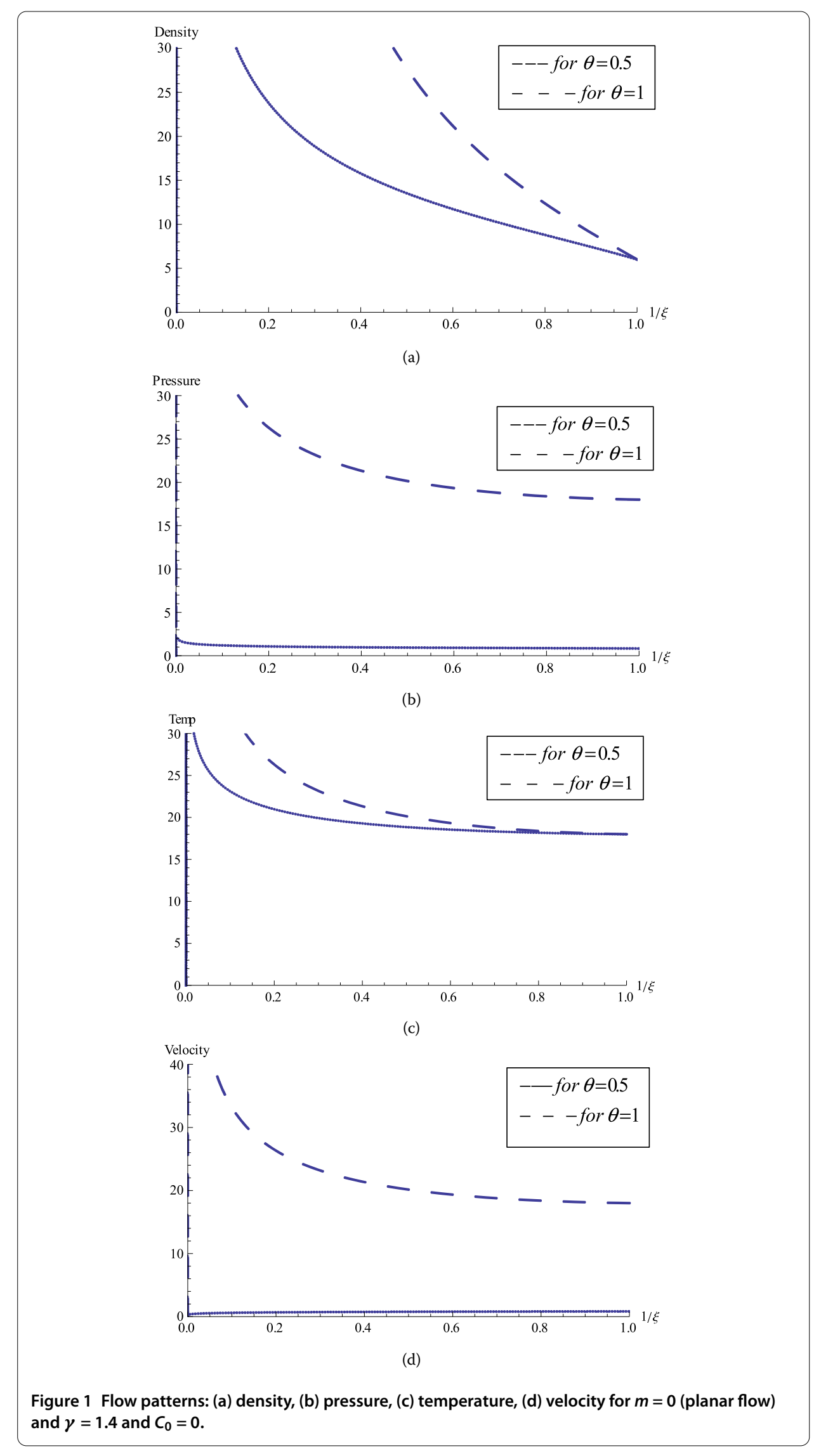




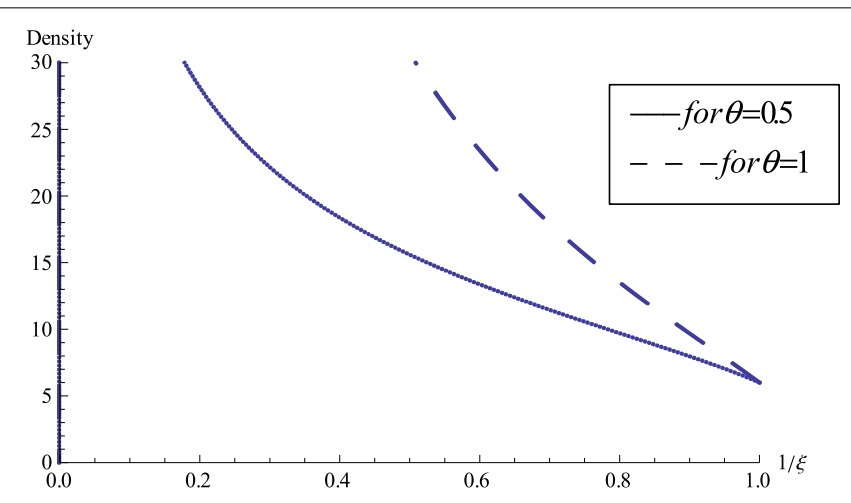

(a)

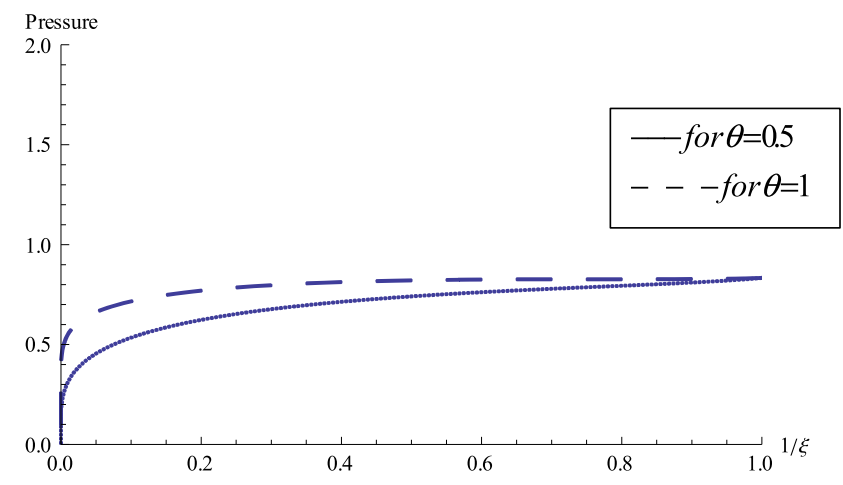

(b)

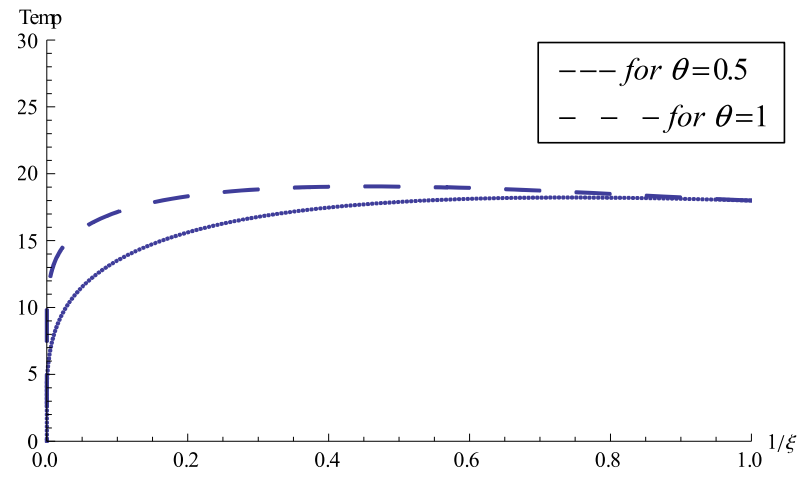

(c)

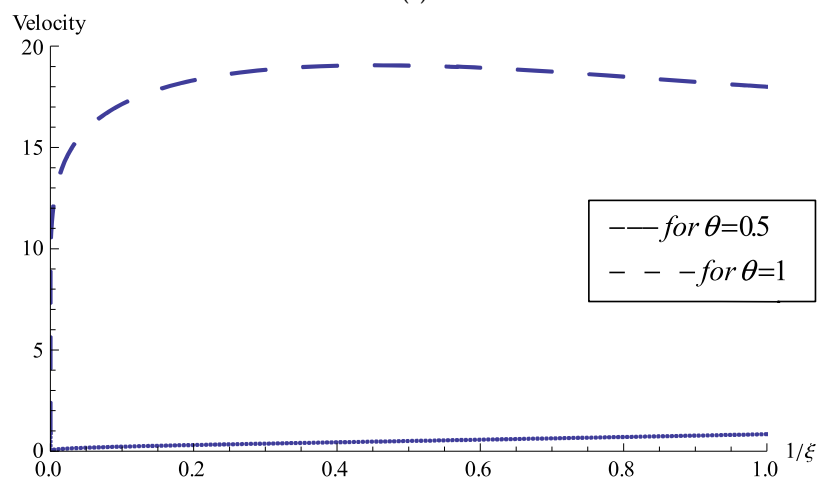

(d)

Figure 2 Flow patterns: (a) density, (b) pressure, (c) temperature, (d) velocity for $m=1$ (cylindrically symmetric flow) and $\gamma=1.4$ and $C_{0}=0$. 
The typical flow profiles show that the density, pressure, temperature and velocity increase behind the shock wave with the increase in the value of $\theta$; this is because a gas particle passing through the shock is subjected to a shock compression. Indeed, this increase in pressure and density behind the shock may also be attributed to the geometrical convergence or area contraction of the shock wave. Figures 1 and 2 show that the growth of the flow variables is slower in cylindrical symmetry as compared with that in planar symmetry. Figures 1 and 2 also confirm the generation of higher pressure near the axis of symmetry, i.e., near $\xi=\infty$. The difference between flow profiles in cylindrical waves and those in planar waves is attributed to the fact that for planar waves, the flow distribution is relatively less influenced by the interaction between the gasdynamic phenomena as compared to cylindrical waves.

\section{Conclusions}

In the present investigation a self-similar method is used to study the flow pattern behind an exponential shock driven by a piston in ideal magnetogasdynamics. The general behavior of density, velocity and pressure profiles remains unaffected due to presence of magnetic field in ideal gas. However, there is a decrease in values of density, velocity and pressure in the case of magnetogasdynamics as compared to non-magnetic case. It may be noted that the effect of magnetic field on the flow pattern is more significant in the case of isothermal flow as compared to that of adiabatic flow.

Competing interests

The authors declare that they have no competing interests.

Authors' contributions

All authors contributed equally to the writing of this paper. All authors read and approved the final manuscript.

\section{Author details}

'Department of Applied Science and Engineering, Indian Institute of Technology Roorkee, Saharanpur Campus, Saharanpur, UP 247001, India. ²Department of Mathematics, Zakir Husain Delhi College, University of Delhi, Delhi, Delhi 110002, India.

Received: 9 May 2013 Accepted: 27 May 2014 Published online: 11 July 2014

\section{References}

1. Hunter, C: Similarity solutions for the flow into a cavity. J. Fluid Mech. 15, 289-305 (1963)

2. Guderley, G: Starke kugelige und zylindrische Verdichtungsstosse in der Nahe des Kugelmittelpunktes bzw. der Zylinderachse. Luftfahrt-Forsch. 19, 302-312 (1942)

3. Greifinger, C, Cole, JD: Similarity solutions for cylindrical magnetohydrodynamic blast waves. Phys. Fluids $\mathbf{5}$, 1597-1607 (1962)

4. van Dyke, M, Guttmann, AJ: The converging shock wave from a spherical or cylindrical piston. J. Fluid Mech. 120, 451-462 (1982)

5. Sharma, VD, Radha, C: On one-dimensional planar and non-planar shock waves in a relaxing gas. Phys. Fluids 6 , 2177-2190 (1994)

6. Sharma, VD, Radha, C: Similarity solutions for converging shocks in a relaxing gas. Int. J. Eng. Sci. 33, 535-553 (1995)

7. Madhumita, G, Sharma, VD: Propagation of strong converging shock waves in a gas of variable density. J. Eng. Math. 46(1), 55-68 (2003)

8. Pandey, M, Sharma, VD, Radha, R: Symmetry analysis and exact solution of magnetogasdynamic equations. Q. J. Mech. Appl. Math. 61, 291-310 (2008)

9. Sharma, VD, Arora, R: Similarity solutions for strong shocks in an ideal gas. Stud. Appl. Math. 114, $375-394$ (2005)

10. Arora, R, Tomar, A, Singh, VP: Similarity solutions for strong shocks in a non-ideal gas. Math. Model. Anal. 17(3), 351-365 (2012)

11. Arora, R, Siddiqui, MJ, Singh, VP: Similarity method for imploding strong shocks in a non-ideal relaxing gas. Int. J. Non-Linear Mech. 57, 1-9 (2013)

12. Sachdev, PL: Propagation of a blast wave in uniform or non-uniform media: a uniformly valid analytic solution. J. Fluid Mech. 52, 369-378 (1972)

13. Chisnell, RF: An analytic description of converging shock waves. J. Fluid Mech. 354, 357-375 (1998)

14. Singh, LP, Husain, A, Singh, M: An analytical study of strong non-planar shock waves in magnetogasdynamics. Adv. Theor. Appl. Mech. 6, 291-297 (2010) 
15. Singh, LP, Husain, A, Singh, M: An approximate analytical solution of imploding strong shocks in a non-ideal gas through Lie group analysis. Chin. Phys. Lett. 27, 014702 (2010)

16. Bluman, GW, Kumei, S: Symmetries and Differential Equations. Springer, New York (1989)

17. Bluman, GW, Cole, JD: Similarity Methods for Differential Equations. Springer, Berlin (1974)

18. Logan, JD, Perez, JDJ: Similarity solutions for reactive shock hydrodynamics. SIAM J. Appl. Math. 39, $512-527$ (1980)

19. Whitham, GB: Linear and Nonlinear Waves. Wiley-Interscience, New York (1974)

doi:10.1186/s13661-014-0142-2

Cite this article as: Arora et al.: Similarity method for the study of strong shock waves in magnetogasdynamics.

Boundary Value Problems 2014 2014:142.

Submit your manuscript to a SpringerOpen ${ }^{\odot}$ journal and benefit from:

- Convenient online submission

Rigorous peer review

- Immediate publication on acceptance

- Open access: articles freely available online

- High visibility within the field

- Retaining the copyright to your article

Submit your next manuscript at $>$ springeropen.com 